Sonderdruck a us

Archiv der Pharmazie

302. Band, Heft 4, Seite 310-318

Verlag Chemie, GmbH, Weinheim / Bergstr.

W. Wiegrebe und E. Roesel

Unsetzungen des 1,2-Dihydro-2-methyl-papaverinols in verdünnter Essigsäure 
W. Wiegrebe und E. Roesel

\section{Umsetzungen des 1,2-Dihydro-2-methyl-papaverinols in verdünnter Essigsäure*)}

Aus dem Institut für Pharmazeutische Technologie der Technischen Universität Braunschweig

(Eingegangen am 30. August 1968)

1,2-Dihydro-2-methyl-papaverinol (II) liefert bei der Behandlung mit 2proz. Essigsäure 3,4-Dihydro-6,7-dimethoxy-3 - (3,4-dimethoxy- $\alpha$-hydroxy-benzyl)-2-methylisochinoliniumion (IX), 9-Hydroxylaudanosin (I), 6,7-Dimethoxy-2-methyl-isochinolinium-ion (III b)**) und Veratrumaldehyd (IV).

\section{Reactions of 1,2-Dihydro-2-methyl-papaverinol in dilute Acetic Acid}

When 1,2-dihydro-2-methyl-papaverinol (II) is treated with $2 \%$ acetic acid, 3,4-dihydro6,7-dimethoxy-3-(3,4-dimethoxy- $\alpha$-hydroxy-benzyl)-2-methyl-isoquinolinium-ion (IX), 9-hydroxylaudanosin(I), 6,7-dimethoxy-2-methyl-isoquinolinium-ion(IIIb)**) and veratraldehyd (IV) are formed.

Im Zusammenhang mit Untersuchungen über das Verhalten des $\alpha-9-H y d r o x y-$ laudanosins (I) bei der Oxydation und Dehydrierung stellte Kirk ${ }^{1}$ ) 1,2-Dihydro-2methyl-papaverinol (II) her und dehydrierte es nach $K n a b e^{2}$ ). Hierbei fand Kirk einen Verbrauch entspr. 1,05 Äquivalenten $\mathrm{Hg}$. Diesen geringen Verbrauch erklärt er folgendermaßen :II wird unter den Versuchsbedingungen schon von 2proz. Essigsäure gespalten und aromatisiert. Zum Beweis führte er folgende Experimente durch: er erhitzte II in 2proz. Essigsäure einmal unter Luftzutritt und zum anderen unter Stickstoffbegasung. Im ersten Fall beobachtete Kirk lediglich eine Spaltung in 6,7-Dimethoxy-2-methyl-isochinolinium-Salz (IIIb) und Veratrumaldehyd (IV), während er im zweiten Fall pc weitere Basen fand, ohne sie zu identifizieren.

Schmid und Karrer ${ }^{3}$ ), die erstmals 1,2-Dihydro-2-methylisochinoline durch Reduktion der entsprechenden quartären Ammoniumsalze mit Lithiumaluminiumhydrid herstellten, weisen auf die schon länger bekannte Tatsache hin, daß solche Verbindungen durch Säuren irreversibel verändert werden; Grewe, Krüger und Vangermain $^{4}$ ) äußerten die Vermutung, daß es sich hierbei um eine Disproportionierung handle.

*) Herrn Prof. Dr. G. Schenck zum 65. Geburtstag gewidmet.

**) In allen Formelbildern kennzeichnet der Buchstabe ,,a" an C-6 und C-7 unsubstituierte Isochinolinderivate, der Buchstabe ,b“ 6,7-Dimethoxy-isochinolin-Abkömmlinge.

1) O. Kirk, Dissertation Braunschweig 1964, S. 32.

2) J. Knabe, Arch. Pharmaz. 292, 416 (1959).

3) H. Schmid und P. Karrer, Helv. chim. Acta 32, 960 (1949).

4) R. Grewe, W. Krüger und E. Vangermain, Chem. Ber. 97, 119 (1964). 
Knabe und Mitarb. ${ }^{5-8}$ ) fanden, daß sich 1,2-Dihydro-2-methyl-papaverin (V) bei Einwirkung von Protonen zu einem 6,7-Dimethoxy-2-methyl-3-(3,4-dimethoxybenzyl)-3,4-dihydro-isochinolinium-salz (VI) umlagert.

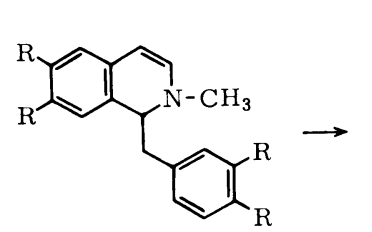

V<smiles>[R]c1ccc(CC2Cc3cc([R])c([R])cc3C=[N+]2C)cc1[R]</smiles>

Vl

$$
\mathrm{R}=\mathrm{OCH}_{3}
$$

Diese Umlagerungsreaktion wurde in der Folge von Dyke und Sainsbury ${ }^{9}$ ) am 6,7-Dimethoxy-2-methyl-1-(3,4-methylendioxy-benzyl)-1,2-dihydro-isochinolin beobachtet.

Bei Versuchen zur Abgrenzung des Geltungsbereiches dieser Umlagerungsreaktion fanden $K$ nabe und Ruppenthal $\left.{ }^{8}\right)^{10}$ ), daß 1,2-Dihydroisochinoline, die in 1-Stellung einen Benzylrest tragen, der Umlagerung unterliegen, während solche, die in 1-Stellung einen Alkyl- oder Phenylrest tragen, in die entsprechenden Tetrahydroisochinoline und Isochinoliniumsalze disproportionieren. Damit war die von Grewe und Mitarb.4 ${ }^{4}$ geäußerte Vermutung bestätigt.

Kirk ${ }^{11}$ ) konnte nachweisen, daß auch C-1 unsubstituiertes 1,2-Dihydro-2-methylisochinolin (VII a) beim Erwärmen in 2proz. Essigsäure in 2-Methyl-1,2,3,4-tetrahydro-isochinolin (VIIIa) und 2-Methyl-isochinolinium-salz (III a) disproportio-

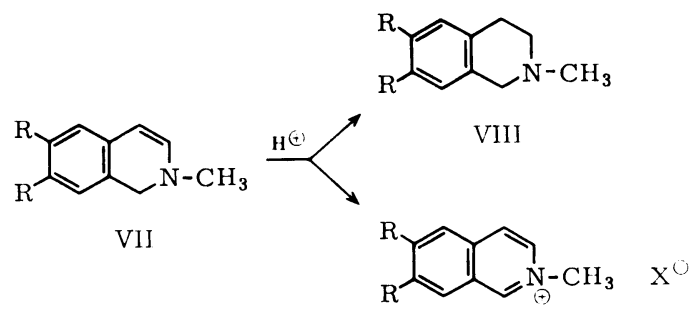

111

$$
\begin{array}{ll}
\text { III, VIl, VIII } & \text { a) } \mathrm{R}=\mathrm{H} \\
\text { b) } \mathrm{R}=\mathrm{OCH}_{3}
\end{array}
$$

$\left.{ }^{5}\right)$ J. Knabe, J. Kubitz und N. Ruppenthal, Angew. Chem. 75, 981 (1963).

6) J. Knabe und J. Kubitz, Arch. Pharmaz. 297, 129 (1964).

$\left.{ }^{7}\right)$ J. Knabe und N. Ruppenthal, Arch. Pharmaz. 297, 141, 268 (1964).

$\left.{ }^{8}\right)$ J. Knabe und N. Ruppenthal, Arch. Pharmaz. 299, 159 (1966).

$\left.{ }^{9}\right)$ S. F. Dyke und M. Sainsbury, Tetrahedron Letters $1964,1545$.

$\left.{ }^{10}\right) J$. Knabe und N. Ruppenthal, Naturwissenschaften 51, 482 (1964).

11) O. Kirk, Dissertation Braunschweig 1964, S. 37. 
niert. Diese Disproportionierung C-1 unsubstituierter Isochinoline konnte Roesel ${ }^{12}$ ) beim 6,7-Dimethoxy-1,2-dihydro-2-methyl-isochinolin (VIIb) bestätigen.

Wird am Benzylrest in p-Stellung ein elektronensaugender Substituent eingeführt, z. B. Brom, so lagert sich das 1-p-Brombenzyl-1,2-dihydro-6,7-dimethoxy-2-methyl-isochinolin nur zum Teil um. Wie Knabe und Ruppenthal ${ }^{8}{ }^{13}{ }^{13}$ fanden, tritt daneben eine Disproportionierung ein, die zu einem Isochinoliniumsalz und einem Tetrahydroisochinolin-Derivat führt.

Die Ergebnisse der vorstehend zitierten Arbeiten machten es unwahrscheinlich, daß bei der Einwirkung von 2proz. Essigsäure auf 1,2-Dihydro-2-methyl-papaverinol (II) ausschließlich Spaltung der Molekel in IIIb und IV erfolgt. Wir hielten es für möglich, daß auch II eine Umlagerung erleidet und daneben noch disproportioniert. Deshalb griffen wir den Versuch von Kirk auf, um das vermutete Umlagerungsprodukt IX zu isolieren und seine Identität durch Vergleich mit synthetisch hergestellter Substanz zu beweisen.

Nach der gegebenen Vorschrift ${ }^{14}$ ) stellten wir II analog ${ }^{3}$ ) her und erwärmten es in 2proz. Essigsäure auf dem Wasserbad. Nach dem Erkalten wurde die gelbe Reaktionslösung aufgearbeitet:

Die saure Reaktionslösung wurde mit Äther ausgeschüttelt. Im DC konnte Veratrumaldehyd (IV) nachgewiesen werden. Zur Identifizierung wurde das IV-2,4Dinitro-phenylhydrazon hergestellt. Der Mischschmelzpunkt mit authentischem Material zeigte keine Depression.

Anschließend wurde die Reaktionslösung mit Ammoniak auf $\mathrm{pH} 8$ eingestellt und mit Äther ausgeschüttelt. Im DC wurde 9-Hydroxylaudanosin (I) (Fleck 2 in Abb. 1; Fleck 1 = authentisches I) gefunden.

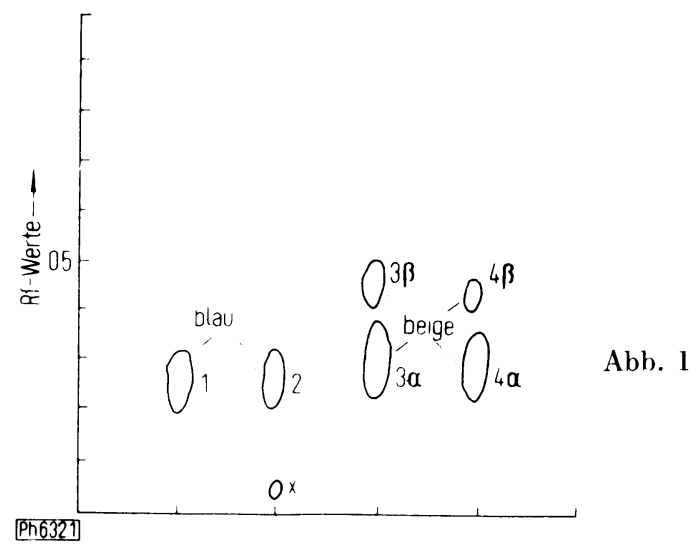

12) $E$. Roesel, unveröffentlichte Versuche, Braunschweig 1964.

13) N. Ruppenthal, Dissertation Braunschweig 1964, S. 37.

14) O. Kirk, Dissertation Braunschweig 1964, S. 75. 
Um auszuschließen, daß das so gefundene I bei der Reduktion des Papaverinol2-methyljodids (X) als Verunreinigung des 1,2-Dihydro-2-methyl-papaverinols (II) entstanden war, wurde II chromatographisch untersucht: es erwies sich als frei von I.

In einem Aliquot des Ätherauszuges wurde 9-Hydroxylaudanosin (I) von der nicht weiter untersuchten Begleitbase (Abb. 1, Fleck $\mathrm{x}$ ) durch präparative Schichtchromatographie abgetrennt und mit $0,1 \mathrm{n} \mathrm{HCl}$ eluiert. Das UV-Spektrum ist ein typisches 1,2,3,4-Tetrahydro-isochinolın-spektrum und deckt sich mit dem Spektrum von authentischem I ( $\left.\lambda_{\max }^{\mathrm{HCl}}: 232 ; 280 \mathrm{~nm}\right)$.

Ein weiterer Teil des o. a. Ätherauszuges wurde zur Trockne gebracht und der Rückstand aus Äthanol kristallisiert. Die erhaltenen Kristalle (Schmp. 136-138') zeigten beim Mischschmelzpunkt mit authentischem $\mathrm{I}^{15}$ ) keine Depression.
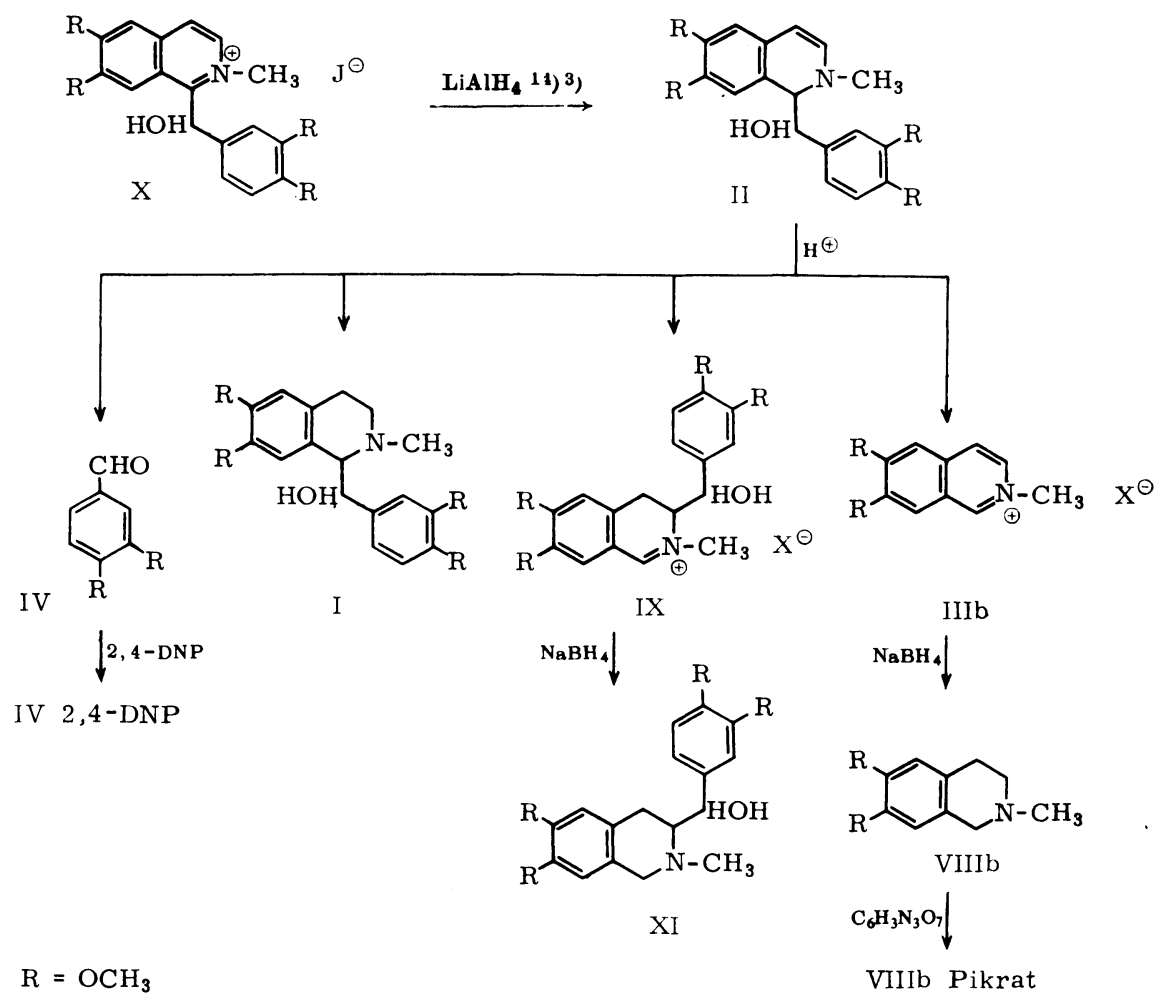

$\left.{ }^{15}\right)$ F. E. King, Ph. L'Ecuyer und F. L. Pyman, J. chem. Soc. (London) 1936, 731. 
Auf Zusatz von Kaliumcyanidlösung zu der hydrogencarbonatalkalischen Reaktionslösung entstand eine Trübung, die mit Äther ausgeschüttelt wurde. Das UVSpektrum des Rückstandes der ätherischen Lösung in $0,1 \mathrm{n} \mathrm{HCl}$ deutet auf das Vorhandensein einer 3,4-Dihydro-2-methyl-isochinoliniumverbindung hin (Abb. 2).

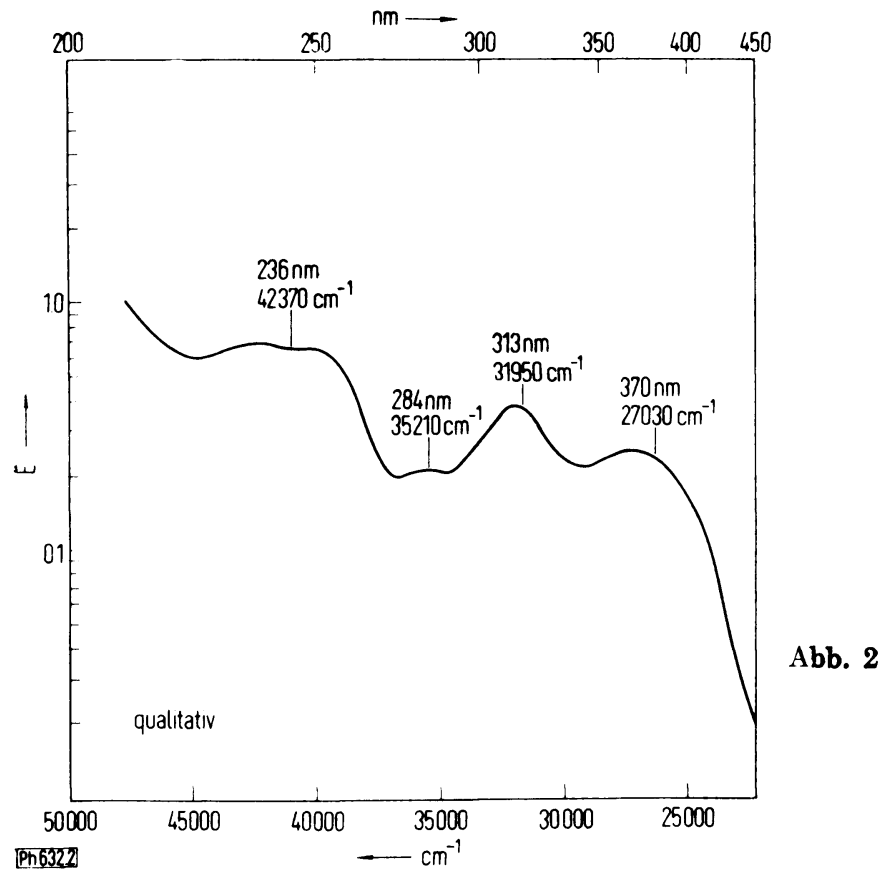

Das Pseudocyanid wurde zerlegt, die 3,4-Dihydro-2-methyl-isochinoliniumverbindung in Äthanol aufgenommen und mit Natriumborhydrid reduziert. Das UVSpektrum $\left(\lambda_{\max }^{\mathrm{HCl}}: 230,278 \mathrm{~nm}\right)$ ist ein typisches 1,2,3,4-Tetrahydroisochinolinspektrum und identisch mit dem UV-Spektrum des 6,7-Dimethoxy-3-(3,4-dimethoxy$\alpha$-hydroxy-benzyl)-2-methyl-1,2,3,4-tetrahydro-isochinolin (XI).

Im DC des Reduktionsansatzes zeigen sich 2 Flecke (Abb. $1 ; 3 \alpha, 3 \beta): 3 x$ hat etwa den Rf-Wert des 9-Hydroxylaudanosins (I) (Abb. 1; 1), $3 \beta$ hat einen höheren. Bei der Detektion mit Formaldehyd-Schwefelsäure färben sich beide Flecke beige, während I sich blau färbt. Das beweist, daß das Reduktionsprodukt des 3,4-Dihydro-2-methyl-isochinoliniumsalzes nicht mit 9-Hydroxylaudanosin (I) identisch ist. Die Flecke $3 \alpha$ und $3 \beta$ entsprechen nach den Rf-Werten auch nicht dem 6,7Dimethoxy-2-methyl-1,2,3,4-tetrahydroisochinolin (VllIb) (Abb. 5; Fleck 5). Wir hielten es daher für möglich, daß das erwähnte 3,4-Dihydroisochinoliniumsalz das gesuchte Umlagerungsprodukt IX mit dem Veratrylrest an C-3 ist. Die Struktur 
des hydrierten Umlagerungsproduktes XI wurde durch Synthese bewiesen, über die wir gesondert berichten werden.

Im DC liefert synthetisches XI ebenso wie das aus der Umlagerung erhaltene XI 2 Flecke (Abb. 1; $4 \alpha, 4 \beta$ ) und färbt sich bei der Detektion mit FormaldehydSchwefelsäure ebenfalls beige. Das Auftreten zweier Flecke im DC läßt sich auf das Vorhandensein von Diastereomeren zurückführen.

Bei der Massenspektroskopie zeigen das durch Synthese gewonnene und das aus der Umlagerung mit nachfolgender Hydrierung erhaltene XI identisches Fragmentierungsverhalten. Das Molekularion $\left(\mathrm{M}^{+}\right)$mit der Masse 373 ist erwartungsgemä $\mathbb{B}$ nur mit etwa $0,1 \%$ rel. Häufigkeit vorhanden. Das häufigste Fragment (100\% rel. Häufigkeit) mit der Masse 206 ist das 1,4-Dihydro-2-methyl-isochinolinium-Ion, während der Benzylrest (m/e 165) nur mit etwa $10 \%$ rel. Häufigkeit auftritt (Abb. 3 und 4).
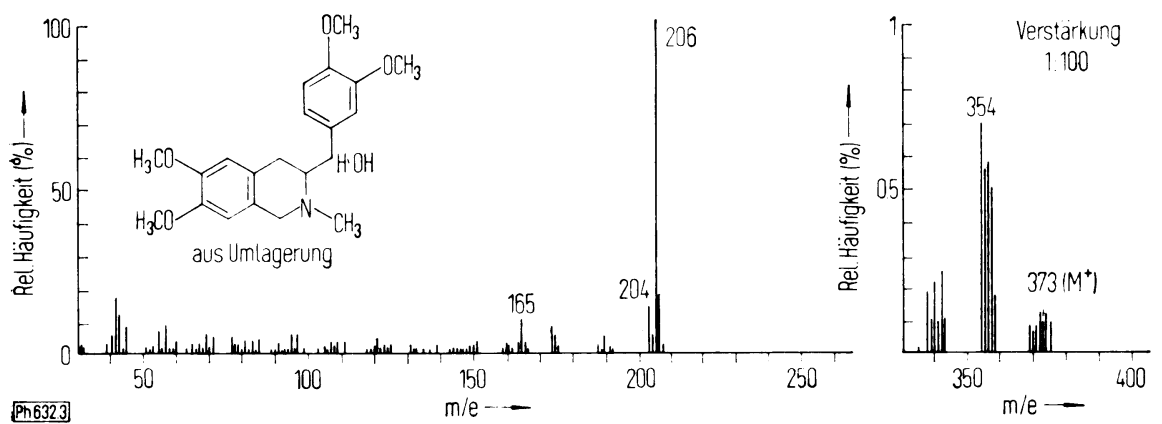

Abb. 3
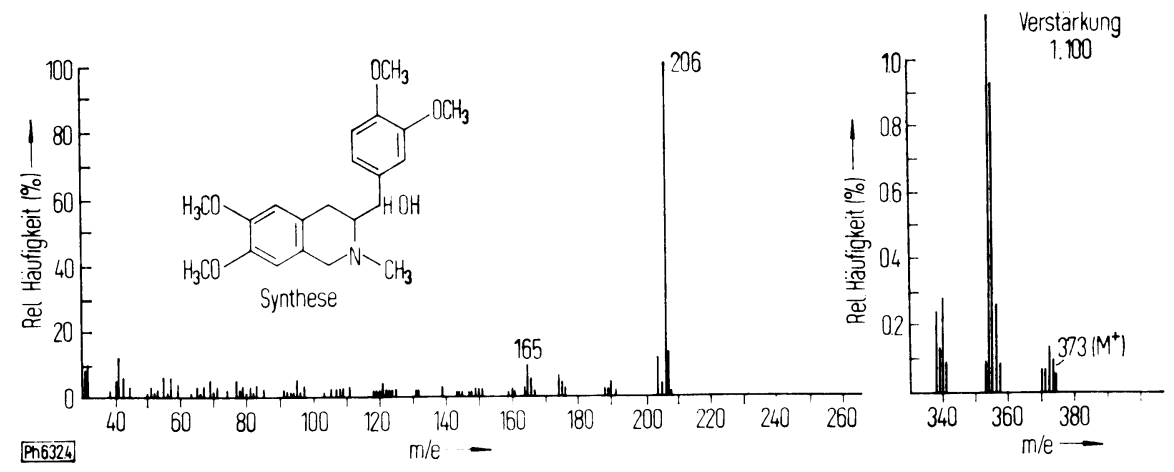

Abb. 4 
Es gelang uns nicht, XI zu kristallisieren. Die Eigenschaften dieses Stoffes und sein Fragmentierungsverhalten werden im Zusammenhang mit der o. a. Synthese beschrieben.

In der wieder angesäuerten wäßrigen Phase wurden die aromatischen Verbin-

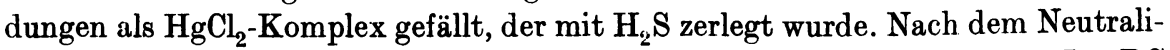
sieren wurde die äthanolische Lösung mit Natriumborhydrid reduziert. Im DC können im wesentlichen zwei Verbindungen festgestellt werden: 6,7-Dimethoxy2-methyl-1,2,3,4-tetrahydro-isochinolin (VIII b) (Abb.5, Fleck 5) und die Substanz mit der wesentlich höheren Rf-Wert (Abb. 5, Fleck y), die nicht näher untersucht wurde.

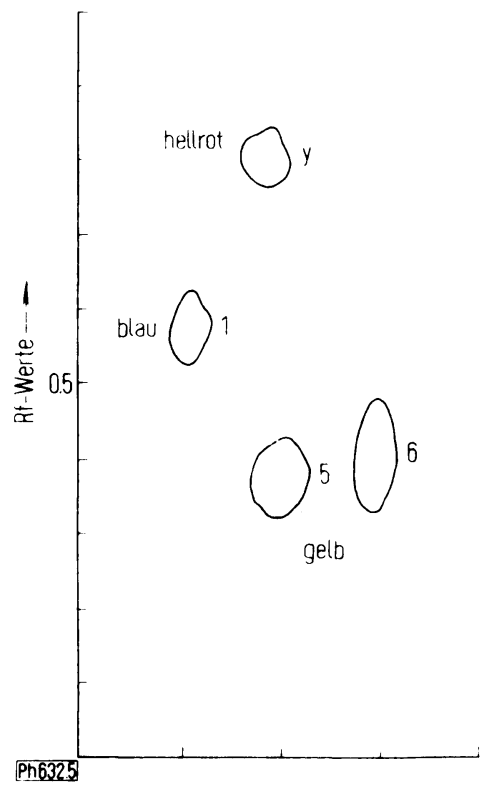

Abb. 5

Es entsprechen 1 authentischem 9-Hydroxylaudanosin (I) und 6 authentischem 6,7-Dimethoxy2-methyl-1,2,3,4-tetrahydro-isochinolin (VIIIb), die Farben, die neben den Flecken angegeben sind, treten bei der Detektion mit FormaldehydSchwefelsäure auf.

Aus der Reduktionslösung wurde VIII b-Pikrat voin Schmp. $157-159^{\circ}$ erhalten. Der Mischschmelzpunkt mit authentischem Material zeigte keine Depression.

Diese Untersuchungen haben unsere Vermutung bestätigt, daß 1,2-Dihydro2-methyl-papaverinol (II) bei der Einwirkung von Protonen hauptsächlich in zwei Richtungen reagiert:

1. Aus II entsteht durch Umlagerung nach $K n a b e^{5}$ ) IX.

2. Das Auftreten der Verbindungen I, TIIL und IV läßt sich folgendermaßen formulieren: 


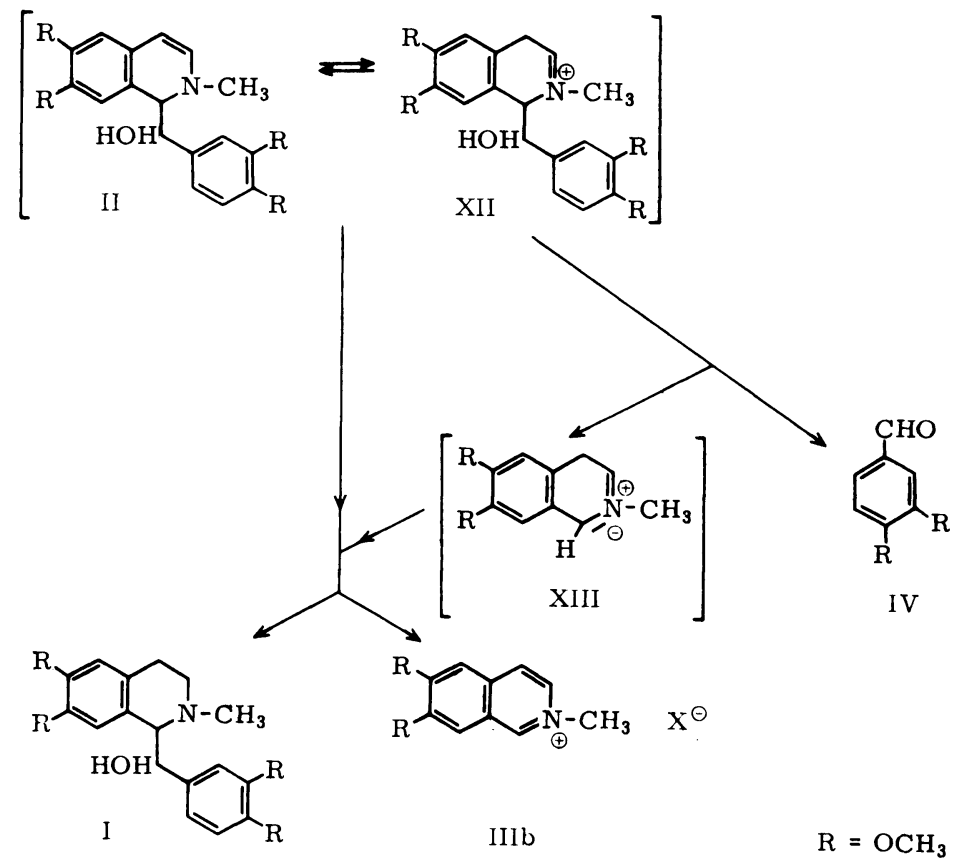

XII, das im Gleichgewicht mit II steht, zerfällt in IV und die Imoniumverbindung XIII, die mit II bzw. XII zu 9-Hydroxylaudanosin (I) und dem 6,7-Dimethoxy-2-methylisochinoliniumion IIIb reagiert. Darüber hinaus muß angenommen werden, daß neben den beschriebenen noch weitere Reaktionen ablaufen.

\section{Beschreibung der Versuche}

1. 1,2-Dihydro-2-methyl-papaverinol (II) $\left.{ }^{14}\right)^{3}$ )

$\mathrm{X}$ liefert durch Reduktion mit Lithiumaluminiumhydrid in absol. Äther II in weißen Nadeln vom Schmp. 130-132 ${ }^{\circ}$, Lit.-Schmp. 135 ${ }^{\circ}$ ); $\lambda_{\max }^{\mathrm{MeOH}}: 283 ; 335 \mathrm{~nm}$.

2. Behandlung von II mit 2 proz. Essigsäure

$300 \mathrm{mg}$ II wurden in $20 \mathrm{ml}$ 2proz. Essigsäure $2 \mathrm{Std}$. auf dem Wasserbade erwärmt.

a) Nicht basische Fraktion

Nach dem Erkalten wurde die gelbe Lösung mit Äther ausgeschüttelt und der über Natriumsulfat getrocknete Åther eingeengt. Im Äther wurde de IV nachgewiesen. Sorptionsschicht: Kieselgel $\mathrm{HF}_{254}$; Fließmittel: Benzol 1, Essigsäureäthylester 1; Laufstrecke: $10 \mathrm{~cm}$; Detektion: UV-Licht $254 \mathrm{~nm}$, Formaldehyd-Schwefelsäure, 30 Min. auf $120^{\circ} \mathrm{er}$ wärmen.

Der Äther wurde abdestilliert, der Rückstand in Äthanol aufgenommen und das IV-2,4Dinitrophenylhydrazon gefällt. Schmp.: 265-266 ${ }^{\circ}$; Lit.-Schmp.: $\left.265^{\circ}{ }^{16}\right)$. Der Mischschmp. mit authentischem Material ergab keine Depression.

16) $R$. L. Shriner et al., The Systematic Identification of Organic Compounds, 4. Auflage, John Wiley \& Sons, Inc., New York. 


\section{b) Fraktion der tertiären Basen}

Die Reaktionslösung wurde mit Ammoniak auf pH 8 eingestellt und mit Äther ausgeschüttelt. Im getrockneten und eingeengten Äther wurde dc I nachgewiesen (Abb. I; Fleck 2). Sorptionsschicht: Kieselgel $\mathrm{HF}_{254}$; Fließmittel: Aceton 60, Methanol 25, Wasser 15; Laufstrecke: $10 \mathrm{~cm}$; Detektion: UV-Licht $254 \mathrm{~nm}$, Formaldehyd-Schwefelsäure, 30 Min. auf $120^{\circ}$ erwärmen.

Ein Teil des Ätherauszuges wurde auf eine Platte mit einer 0,2-mm-Schicht aus Kieselgel $\mathrm{PF}_{254}$ aufgetragen und diese einmal $15 \mathrm{~cm}$ mit Aceton 60, Methanol 25, Wasser 15 entwickelt. Unter dem UV-Licht wurde das Band von I markiert. Nach dem Abheben wurde mit 0,1 n $\mathrm{HCl}$ eluiert und das UV-Spektrum gemessen.

c) Pseudocyanid-Fraktion

Die wäßrige Phase von b) wurde schwach angesäuert, hydrogencarbonatalkalisch gemacht und mit einer konz. Kaliumcyanidlösung versetzt. Die entstandene Trübung wurde mit Äther ausgeschüttelt.

Der getrocknete Äther wurde abgedampft, das Pseudocyanid mit einigen Tropfen verd. Essigsäure zerlegt, IX in $5 \mathrm{ml}$ Äthanol aufgenommen und mit $100 \mathrm{mg}$ Natriumborhydrid 1 Std. auf dem Wasserbad erwärmt. Nach 30 Min. wurden weitere $100 \mathrm{mg}$ Natriumborhydrid zugegeben. Nach dem Erkalten wurde der Utberschuß an Natriumborhydrid mit Eisessig zerstört, die Lösung i. Vak. auf einige $\mathrm{ml}$ eingeengt, mit $10 \mathrm{ml}$ Wasser versetzt und nach dem Alkalisieren mit Äther ausgeschüttelt. Der getrocknete Äther wurde eingeengt. DC: Abb. 1; Sorptionsschicht usw. wie unter b).

Ein Teil des Äthers wurde mit 5 mg ausgeglühter Kohle versetzt und i. Vak. zur Trockne gebracht. Von dem an die Kohle adsorbierten XI wurde ein Massenspektrum gemessen, Abb. 3.

d) Aromaten-Fraktion

Die wäßrige Phase von c) wurde mit Salzsäure angesäuert, HCN und Äther wurden i. Vak. entfernt. Dann wurde mit $\mathrm{HgCl}_{2}$-Lösung versetzt, der Niederschlag abgesaugt, mit Äthanol angeschlämmt und unter Zusatz von Salzsäure mit $\mathrm{H}_{2} \mathrm{~S}$ zerlegt. Ein Teil der äthanol. Lösung wurde i. Vak. zur Trockne gebracht und vom Rückstand in $0,1 \mathrm{n} \mathrm{HCl}$ ein UV-Spektrum gemessen, $\lambda_{\max }: 253 ; 282 ; 314 \mathrm{~nm}$. Die äthanol. Lösung wurde wie unter c) beschrieben mit Natriumborhydrid reduziert und aufgearbeitet. Nach dem Abdampfen des getrockneten Äthers wurde in absol. Äthanol aufgenommen und mit äthanol. Pikrinsäurelösung versetzt. Das ausgefallene VIIIb-Pikrat wurde aus absol. Äthanol umkristallisiert. Schm.: 157-159 ${ }^{\circ}$. Lit.-Schmp.: 159-160 ${ }^{\circ}$ ). Der Mischschmp. mit authentischem VIIIb-Pikrat zeigte keine Depression.

DC Abb. 5; Sorptionsschicht: Kieselgel $\mathrm{HF}_{254}$; Fließmittel: Aceton 60, Methanol 25, Wasser 15, $\mathrm{NH}_{3}$ 25proz. 5 Trpf.; Laufstrecke: $16 \mathrm{~cm}$; Detektion: UV-Licht $254 \mathrm{~nm}$; Formaldehyd-Schwefelsäure, $30 \mathrm{Min}$. auf $120^{\circ}$ erwärmen.

\section{Allgemeine Angaben}

Die Schmp. und Mischschmp. wurden auf dem Kofler-Mikroheiztisch bestimmt und sind unkorrigiert. Die UV-Spektren wurden im Zeiss-Spektralphotometer PM Q II gemessen. Die Massenspektren wurden mit dem Gerät MS 9 der Firma AEI aufgenommen.

Herrn Prof. Dr. Awe + danken wir für ausführliche Diskussionen; Herrn Prof. Dr. Dr. h. c. H. H. Inhoffen und Herrn Doz. Dr. H. Budzikiewicz sind wir für die Massenspektren dankbar.

$\left.{ }^{17}\right)$ F. L. Pyman, J. chem. Soc. (London) 95, 1274 (1909). 